\title{
Extended pleurectomy and decortication: Video atlas of operative steps
}

\author{
R. Taylor Ripley, MD, and Nihanth Palivela, BS, Houston, Tex
}

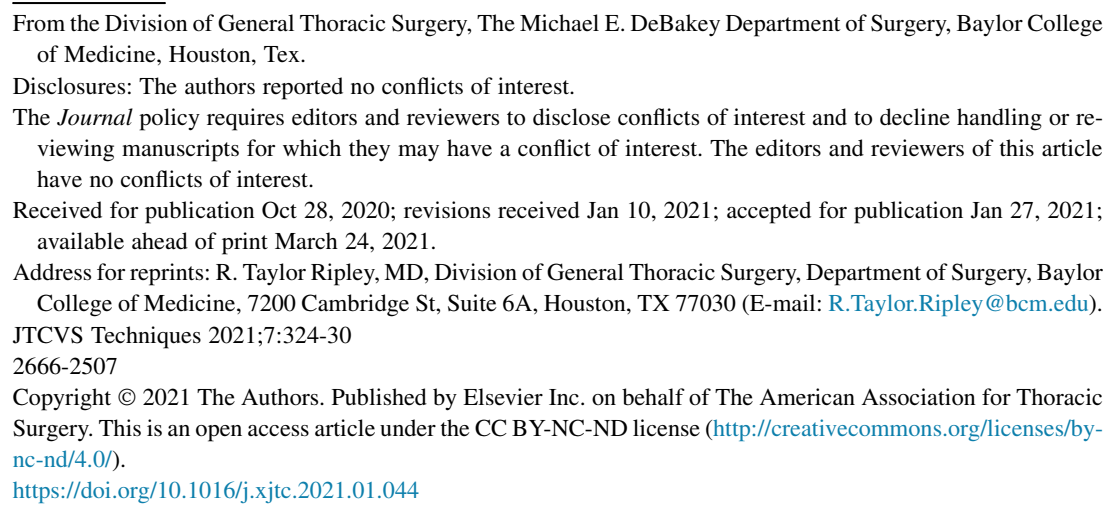
Video clip is available online.

Feature Editor Note-Video Atlas Articles (VAAs) are peer-reviewed descriptions of an operative procedure distilled into a series of steps, with each step taught to the reader through its own narrated video and short corresponding text. The purpose of the VAA is to provide continuing surgical education in a format that is qualityassured, easily accessible, and high impact. Much of the excitement around our new series of VAAs surrounds robotic and thoracoscopic/laparoscopic procedures. Our field has progressed rapidly into one in which robotic and thoracoscopic surgery have improved clinical outcomes, and we drive much of the innovation in minimally invasive surgical platforms and technique. The ability to record highresolution video during the routine conduct of robotic and thoracoscopic/laparoscopic surgery has facilitated this opportunity in structured, peer-reviewed, expert instruction of index cases in thoracic surgery and is the bedrock for the VAA initiative. Open surgery, however, has become less frequent in our training programs, and operations that are performed open are now more likely to be complex rather than routine cases. This highlights a gap in surgical training that can be narrowed with novel methods of continuing surgical education. In this VAA, the authors describe an extended pleurectomy/decortication procedure for malignant pleura mesothelioma in a structured and comprehensive series of 12 steps. High-resolutions videos recorded surgery.

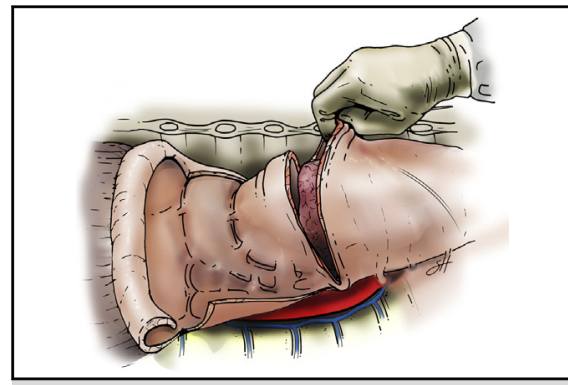

Removal of malignant pleural mesothelioma by extended pleurectomy and decortication.

\section{CENTRAL MESSAGE \\ The operative steps for an extended pleurectomy and decortication are described with a video atlas. An ePD can achieve a macroscopic complete resec- tion for MPM with preservation of the lung.}

See Commentary on page 331.

from a surgeon's headlight camera are provided for each step and allow the reader to experience the conduct of each step of this complex, open operation from the surgeon's perspective. This is the first VAA of an open procedure and showcases the potential of this format to advance continuing surgical education for open thoracic

\section{Bryan M. Burt, MD}

Malignant pleural mesothelioma (MPM) is the most common type of malignant mesothelioma, which arise from the pleural, peritoneum, pericardium, and tunica vaginalis. The incidence of MPM is about 3000 patients per year in the United States. The etiology of the majority of cases is asbestos exposure. Surgery with chemotherapy results in long-term survival in some patients. ${ }^{1}$ Surgery has a role in the diagnosis, staging, and treatment, with both palliative and curative intents. ${ }^{2}$ The National Comprehensive Cancer Network, the European Society for Medical Oncology, and American Society of Clinical Oncology have guidelines 
that recommend surgery for MPM for both diagnosis and treatment. ${ }^{3-5}$

A macroscopic complete resection is the goal of curative-intent surgery. ${ }^{6,7}$ Negative microscopic margins (R0 resection) are not feasible since MPM covers all surfaces of the thoracic cavity and resection of these structures is not possible. The National Cancer Institute, the International Association for the Study of Lung Cancer, and the Mesothelioma Applied Research Foundation have helped standardize the operations for MPM. To achieve a macroscopic complete resection, either a lungsparing surgery called an extended pleurectomy/decortication (ePD) or a lung-sacrificing surgery called an extrapleural pneumonectomy is performed. ${ }^{8}$ A visceral and parietal pleurectomy includes the "extended" descriptor when resection of the diaphragm and/or pericardium are performed. Whether a partial diaphragm resection should be included as an ePD is questionable, but despite differences in the operations, the goal of an ePD is to remove all visible, palpable, or viable tumor while sparing the lung.

This article outlines the steps to perform an ePD. This approach is surgeon-specific, and alternatives to most aspects of this operation exist. Extensive training is necessary before performing this operation independently.

\section{PREOPERATIVE EVALUATION}

An extensive evaluation is necessary to determine whether a patient is an operative candidate. The evaluation includes diagnostic and subtype confirmation, establishment of clinical stage, and assessment of cardiopulmonary status. Patients are evaluated for a treatment plan that may include surgery, chemotherapy, immunotherapy, radiotherapy, palliation, and clinical trial eligibility. Presentation at multidisciplinary tumor board helps finalize recommendations.

Video-assisted thoracoscopic surgery (VATS) or computed tomography (CT)-guided biopsies establish the diagnosis. Confirmation of the diagnosis or subtyping requires rebiopsy or review of the pathology once referred to a center specializing in MPM. Repeated VATS biopsies may be required. After diagnosis, positron emission tomography scans, CT scans, and occasionally magnetic resonance imaging help establish clinical staging. The positron emission tomography scan evaluates the thoracic cavity, mediastinal lymph nodes, and potential metastatic sites. Magnetic resonance imaging assists with identifying local invasion but does not contribute significantly more than CT.

Staging with mediastinoscopy and diagnostic laparoscopy helps to identify a subset of patients with advanced disease that is not noted on imaging. ${ }^{9,10}$ Diagnostic laparoscopy reveals disease in $17 \%$ of patients. Mediastinoscopy may exclude marginal operative candidates, but it does not fully evaluate lymph node metastases because pleural disease can spread to nodes other than the peritracheal nodes. Repeated VATS biopsies, mediastinoscopy, and diagnostic laparoscopy can be performed during the same operation.

Assessment of cardiopulmonary status is necessary to lower perioperative risk. Pulmonary function tests and ventilation/perfusion nuclear scans are performed. Postoperative predicted pulmonary volumes are calculated to determine eligibility for an extrapleural pneumonectomy even when an ePD is planned. The diffusion-limited carbon monoxide is important to identify restricted pulmonary diffusion that occurs with asbestosis. An absolute threshold for pulmonary function tests or ventilation/perfusion values in which an ePD should not be performed is debatable; however, when the numbers are particularly low, the lung will likely not regain significant function. An echocardiogram and an electrocardiogram are performed. Right heart catheterization may be necessary, given that echocardiogram does not adequately estimate right heart pressure; however, cardiology consultation is advisable for most patients especially with comorbidities.

\section{OPERATIVE APPROACH}

\section{Preoperative Planning and Positioning}

An epidural catheter, arterial line, central venous catheters, nasogastric tube, and urinary catheter are placed. A pulmonary artery catheter is rarely necessary. A central venous catheter is inserted in the internal jugular vein; however, femoral vein access is an acceptable alternative. Blood loss is normally 300 to $500 \mathrm{~mL}$. Injury to vessels such as the superior vena cava may result in larger-volume blood loss, and femoral access may be helpful for rapid resuscitation. Epidural catheters may be eliminated and regional anesthetic agents used, which may prevent exacerbation of hypotension from unilateral sympathectomy. A flexible bronchoscopy is unnecessary; therefore, a double-lumen endotracheal tube is placed to start. Two-lung ventilation is maintained as long as possible to decrease barotrauma, including during the initial pleurectomy. After the operation, the double-lumen tube is changed to a single-lumen tube and a therapeutic flexible bronchoscopy is performed to remove secretions. The operation is performed in standard lateral decubitus position with slight anterior rotation of the hip.

\section{Operative Steps}

Step 1. Video 1 and 2: Incision and development of the extrapleural plane. An extended posterolateral thoracotomy is performed with the incorporation of previous VATS port sites if feasible. The latissimus dorsi and serratus anterior are mobilized and spared. The sixth rib is removed with a subperiosteal dissection to spare the intercostal muscles, which are approximated during closing. The extrapleural space is developed circumferentially from the resected 
rib. The incision is extended once lack of chest wall invasion is determined. Hemostasis is continually obtained with packing areas while working elsewhere and coagulation with Aquamantys Bipolar Sealers (Medtronic, Minneapolis, Minn) or argon plasma coagulation (instrument list: Table 1 and Table E1).

Step 2. Video 3: Anterior extrapleural dissection. The anterior pleural dissection removes the parietal pleural from the chest wall into the pericardiosternal recess. Internal mammary and pericardial fat pad nodes are removed. When the dissection reaches the pericardium, the pleura is peeled off the fibrous pericardium to the pulmonary veins if the disease does not invade the pericardium. If the disease invades the pericardium, separation of the fibrous and serous pericardium may complete the dissection. Alternatively, the anterior dissection is stopped and the pericardium is resected later in the operation.

Step 3. Video 4: Apical extrapleural dissection. The apical pleural dissection removes the disease from the chest wall and apex. Dissection is continued along the mediastinal pleura by pushing the structures into the mediastinum to avoid traction injuries to the recurrent laryngeal nerves, the lymphatics, vessels, and airways. Once the azygous vein (aortic arch/left side) is identified, this dissection is completed. The apical dissection will connect to the anterior dissection at the superior vena cava.

Step 4. Video 5: Posterior extrapleural dissection. The posterior pleural dissection is developed from the chest wall over the azygous and the esophagus to posterior hilum. During left-sided operations, the pleurectomy continues over

TABLE 1. Unique instrumentation for extended pleurectomy and decortication

\begin{tabular}{ll}
\hline \multicolumn{1}{c}{ Instrument } & \multicolumn{1}{c}{ Company } \\
\hline $\begin{array}{l}\text { Matson Rib Double Ended Elevator and } \\
\text { Stripper Raspatory }\end{array}$ & Novo Surgical Inc \\
Stille-Luer-Type D/A 10MM Curved Jaws & Cen-Med Enterprises Inc \\
\hline $\begin{array}{l}\text { Alexander Periosteotome- Sharp } \\
\text { Doyen Coastal Elevator: Cobb, Right, and } \\
\text { Left Blade, Heavy Duty Round Handle }\end{array}$ & Superior Instruments \\
\hline $\begin{array}{l}\text { Cobb Elevator } \\
\text { Handpiece Interpulse w/Coaxial High Flow }\end{array}$ & Stryker \\
\hline $\begin{array}{l}\text { Aquamantys 6.0 Bipolar Sealer } \\
\text { Argon Beam Coagulator }\end{array}$ & Medtronic \\
\hline ProTack 5-mm Fixation Device & Valleylab Inc. \\
\hline LIGACLIP Endoscopic Rotating Multiple \\
Clip Applier & Medtronic \\
\hline Carter-Tomason Suture Passer & \\
\hline Gore-Tex Dual Mesh & CooperSurgical \\
\hline 20 cm $\times 30 \mathrm{~cm} \times 1.0$ mm & W. L. Gore \& Associates \\
\hline Endo GIA Ultra Universal Stapler & Covidien \\
\hline
\end{tabular}

the aorta; avoiding a dissection behind the aorta is critical to prevent injury to the segmental branches. Sharp dissection of fibrous adhesions is required to mobilize the parietal pleura to the visceral pleura; complete mobilization facilitates the visceral dissection later. Below the inferior pulmonary vein, the posterior dissection continues to the diaphragm along the inferior mediastinum. The lymph node dissections from levels 7, 8, and 9 are performed.

Step 5. Video 6: Lymph node dissection. The level 7 lymph node dissection is completed. Although not shown, lymph node dissections include levels $3,4 \mathrm{R}, 5,6,8,9,10,11$, 12 , and internal mammary nodes depending on the side of the operation. These are performed during dissections in the respective areas. Lymph nodes within the intercostal muscles are also retrieved.

Step 6. Video 7: Diaphragm resection. Preservation of portions of the diaphragm is feasible depending on extent of disease. Anteriorly, removal of the diaphragmatic disease exposes the angle between the diaphragm and pericardium and, posteriorly, the disease is peeled off above the esophagus on the right and the aorta on the left. Often, resection of the diaphragm is easier from the abdominal side; therefore, the diaphragm is opened at the most lateral point and this opening is extended to the mediastinum while preserving as much muscle as possible. The peritoneum at the base of the diaphragm is scored about 1 to $2 \mathrm{~cm}$ lateral to the mediastinum. The diaphragm medial to this line becomes a cuff to attach the diaphragm patch to prevent herniation.

Step 7. Video 8: Pericardial resection. During the anterior parietal dissection at the point in which the pleura cannot be peeled off the pericardium, it is opened. The pericardiectomy is extended superiorly to the great vessels, inferiorly to the diaphragm, posteriorly to the pulmonary veins, and anteriorly to the pleura of the contralateral lung. Complete transection is easier from an intrapericardial approach. Stay sutures are placed to prevent retraction of the pericardium to the contralateral side with subsequent hemodynamic instability.

Step 8. Video 9: Visceral decortication. The operative lung is inflated and an incision is performed through the pleura; often both the visceral and parietal pleura with the thickened disease are fused. With one hand that provides retraction, the lung is separated from the visceral pleura by both blunt and sharp dissections. The disease may be too adherent for decortication. The lingula or right middle lobe may require resection and wedge resections often facilitate pleurectomy at difficult angles. The fissure is approached by performing the visceral pleural dissection from each lobe to the base of the fissure. With complete fissures, this dissection may require dissection off the pulmonary arteries. Completion of the visceral pleurectomy is obtained when this dissection connects to the parietal pleural dissection. 
Step 9. A: Video 10: Diaphragm reconstruction (part I). A nonabsorbable, 1- to 2-mm patch is placed in the native position at the eighth intercostal space (ICS) anteriorly, ninth ICS laterally, and tenth ICS posteriorly. Alternatively, reconstruction with a biological material such as an acellular dermal matrix may decrease infection rate caused by air leaks. No. 2 polyglactin 910 sutures are sewn through the chest wall, into diaphragm patch as a vertical mattress with the edge everted into the chest cavity, and back through the chest wall one interspace higher. A suture-passing device may help. Sutures to the erector spinae muscles are inserted.

B: Video 11: Diaphragm reconstruction (part II). Once the lateral diaphragm patch is secured, the size and tautness of the patch are based on the sutures along the mediastinum. Anteriorly, a portion of the muscular diaphragm is usually preserved between the chest wall and pericardium which is sewn to the anterior edge of the patch with non-absorbable sutures. Next, the patch is attached to the base of the pericardium, which provides more rigidity to the reconstruction compared with the anterior muscular diaphragm. The medial border of the patch is secured from the pericardium to the residual cuff of the diaphragm. The reconstruction is completed over the vertebral bodies with a ProTack 5-mm fixation device (Medtronic).

Step 10. A: Video 12: Pericardial reconstruction (part I). The pericardial reconstruction prevents herniation and torsion. The pericardium is reconstructed with a $0.1-\mathrm{mm}$ polytetrafluoroethylene patch that is attached with 20 nonabsorbable sutures. Alternatively, polyglactin 910 mesh or bovine pericardium is used, which may decrease infectious complications. Over the great vessels, the pericardium does not need to be closed. The pericardium is reconstructed starting with horizontal mattress sutures in the anterior pericardium that are parachuted into the chest.

B: Video 13: Pericardial reconstruction (part II). The inferior pericardial patch is secured to the confluence of the diaphragm and pericardium through diaphragm patch. Avoiding compression of the inferior vena cava is critical during this reconstruction. The patch is secured to the posterior pericardium along the inferior pulmonary vein (IPV) and superior pulmonary veins. The placement of these sutures will determine the size of the patch, given that the anterior sutures are already in place; if the patch is too tight, restriction may occur. The patch is fenestrated to prevent tamponade.

Step 11. Video 14: Povidone-iodine scrub. Several intraoperative therapies are available that may decrease ipsilateral recurrences. Povidone-iodine is administered to the intrathoracic cavity after complete resection and reconstruction of diaphragm and pericardium with protocol modified from Lang-Lazdunski and colleagues (Table 2). ${ }^{11}$ Other intraoperative adjuncts include chemotherapy, photodynamic
TABLE 2. Procedure for the administration of povidone-iodine

\begin{tabular}{|c|c|}
\hline Step & Procedure \\
\hline 1 & $\begin{array}{l}\text { Prepare: hyperthermic }\left(40^{\circ} \mathrm{C}-41^{\circ} \mathrm{C}\right) 3 \mathrm{~L} \text { of saline with } 10 \% \\
\text { povidone-iodine }\end{array}$ \\
\hline 2 & Scrub incision and chest with $10 \%$ pure povidone-iodine \\
\hline 3 & Allow to bathe for $5 \mathrm{~min}$ in chest \\
\hline 4 & Irrigate and bathe with water for $5 \mathrm{~min}$ \\
\hline \multirow[t]{2}{*}{5} & $\begin{array}{l}\text { Administer } 3 \mathrm{~L} \text { of hyperthermic povidone-iodine with } \\
\text { handpiece }\end{array}$ \\
\hline & Interpulse with Coaxial High Flow (Stryker) \\
\hline 6 & Allow to bathe for $5 \mathrm{~min}$ in chest \\
\hline 7 & $\begin{array}{l}\text { Repeat scrub incision and chest with } 10 \% \text { pure povidone- } \\
\text { iodine }\end{array}$ \\
\hline 8 & Allow to bathe for $5 \mathrm{~min}$ in chest \\
\hline 8 & Irrigate and bathe with water for $5 \mathrm{~min}$ \\
\hline 9 & Assess thoroughly for hemostasis \\
\hline
\end{tabular}

therapy, hydrogen peroxide, and argon beam coagulation. No therapy has proven efficacy or benefit over another.

Step 12. Video 15: Final anatomy. Overview of the anatomy of the residual thoracic cavity after pleurectomy.

\section{Operative Considerations}

- The latissimus dorsi and the serratus anterior can be mobilized without transection. If residual space in the chest results in an empyema or persistent air leaks, these muscles are available as pedicled muscle flaps. This approach requires subcutaneous drain placement to avoid seroma formation. In addition, if the muscle does not seal the chest completely, the subcutaneous space can communicate with the residual thoracic cavity.

- The sixth rib is removed in a periosteal plane. When closing the chest after resection of this rib, the intercostal sutures do not pull the ribs completely together to help decrease postoperative pain. The intercostal bundles are sutured together and the muscle flaps are parachuted into the incision to prevent fluid shift through the chest wall. Alternatively, the seventh rib may be removed for better exposure of the diaphragm.

- Once the rib is removed, chest wall invasion is assessed during the initial pleurectomy to determine resectability. Chest wall biopsy with rapid pathological assessment (frozen section) can help determine whether chest wall invasion is present. However, frozen section only augments gross findings for confirmation.

- Dissection of the pleura off vascular, lymphatic, and nerve tissue is performed by pushing the mediastinum off the pleura rather than pulling the pleura off the mediastinum to decrease traction injuries. 
- Identifying the IPV and performing the pleurectomy from the posterior pleura below the vein to the pericardium anteriorly will help identify the diaphragm to create a cuff for the reconstruction.

- Dissection of the parietal pleural across the hilar vessels onto the pulmonary parenchyma will facilitated completion of the visceral pleurectomy.

- With large tumors, the visceral decortication can be performed before completion of the parietal pleurectomy. This maneuver will facilitate the safe approach to the mediastinum and hilum, especially between the IPV and the diaphragm.

- For thin visceral pleura without gross disease, ablation of the pleura is acceptable if the visceral pleura cannot be adequately decorticated.

- When removing the diaphragm, holes in the peritoneum are closed whenever possible.

- Either avulsing or transecting the diaphragm and removing it from the peritoneal surface is often simpler than resection of the diaphragm from the thoracic cavity secondary to the bulky pleural disease.

- Creation of a cuff of diaphragm along the mediastinum over the aorta and esophagus helps reconstruct the diaphragm patch and prevent herniation. If disease is involved, this area can be decorticated and spared.

- Traction on the phrenic vein is critical to avoid because tearing this vein can track onto the inferior vena cava and into the abdomen.

- During reconstruction of the pericardium, the patch must remain loose and have fenestrations to prevent restriction and tamponade.

- Four chest tubes are placed in anterior, lateral, diaphragm, and posterior positions. Suction on the tubes may result in significant loss of tidal volume secondary to air leaks.

\section{CONCLUSIONS}

Extended pleurectomy and decortication can achieve a macroscopic complete resection of malignant pleural mesothelioma with the preservation of the lung. Outlined herein are the steps to perform this operation.

\section{References}

1. Rusch VW, Giroux D, Kennedy C, Ruffini E, Cangir AK, Rice D, et al. Initial analysis of the international association for the study of lung cancer mesothelioma database. J Thorac Oncol. 2012;7:1631-9.

2. Bueno R, Opitz I, Taskforce IM. Surgery in malignant pleural mesothelioma. J Thorac Oncol. 2018;13:1638-54.

3. Baas P, Fennell D, Kerr KM, Van Schil PE, Haas RL, Peters S, ESMO Guidelines Committee. Malignant pleural mesothelioma: ESMO Clinical Practice Guidelines for diagnosis, treatment and follow-up. Ann Oncol. 2015;26(suppl 5):v31-9.

4. Ettinger DS, Wood DE, Akerley W, Bazhenova LA, Borghaei H, Camidge DR, et al. NCCN guidelines insights: malignant pleural mesothelioma, version 3.2016. J Natl Compr Canc Netw. 2016;14:825-36.

5. Kindler HL, Ismaila N, Armato SG III, Bueno R, Hesdorffer M, Jahan T, et al. Treatment of malignant pleural mesothelioma: American Society of Clinical Oncology Clinical Practice Guideline. J Clin Oncol. 2018;36:1343-73.

6. Rice D, Rusch V, Pass H, Asamura H, Nakano T, Edwards J, et al. Recommendations for uniform definitions of surgical techniques for malignant pleural mesothelioma: a consensus report of the International Association for the study of Lung Cancer International Staging Committee and the International Mesothelioma Interest Group. J Thorac Oncol. 2011;6:1304-12.

7. Ripley RT. Extended pleurectomy and decortication for malignant pleural mesothelioma. Thorac Surg Clin. 2020;30:451-60.

8. Friedberg JS, Culligan MJ, Tsao AS, Rusch V, Sepesi B, Pass HI, et al. A proposed system toward standardizing surgical-based treatments for malignant pleural mesothelioma, from the Joint National Cancer Institute-International Association for the Study of Lung Cancer-Mesothelioma Applied Research Foundation taskforce. J Thorac Oncol. 2019;14:1343-53.

9. Rice DC, Erasmus JJ, Stevens CW, Vaporciyan AA, Wu JS, Tsao AS, et al. Extended surgical staging for potentially resectable malignant pleural mesothelioma. Ann Thorac Surg. 2005;80:1988-92; discussion 92-3.

10. Ripley RT, Palivela N, Groth SS, Choi EA, Cornwell LD, Carrott PW, et al. Diagnostic laparoscopy improves staging of malignant pleural mesothelioma with routine PET imaging. Ann Thorac Surg. December 1, 2020 [Epub ahead of print].

11. Lang-Lazdunski L, Bille A, Belcher E, Cane P, Landau D, Steele J, et al. Pleurectomy/decortication, hyperthermic pleural lavage with povidone-iodine followed by adjuvant chemotherapy in patients with malignant pleural mesothelioma. J Thorac Oncol. 2011;6:1746-52. 


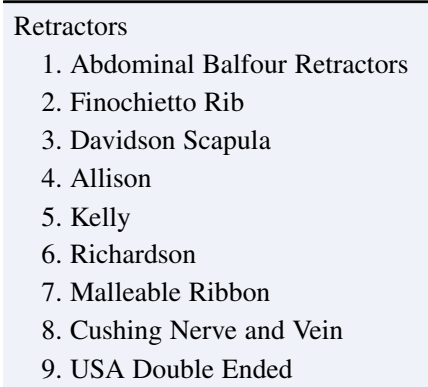

Clamps

1. Mosquito Halsted

2. Artery Crile

3. Hemostatic Crile

4. Rochester-Pean

5. Hemostatic Gemini

6. Classic Rochester-pean

7. Thoracic Mixter

8. Artery Ochsner

9. Tissue Allis 5X6 Teeth

10. Towel Backhaus

11. Sponge Foerster

12. Sponge Holding Serr Jaws

13. Thoracic Willauer-Allis

14. Tissue Thoracic Babcock

15. Judd-Allis

16. Harken CVD DeBakey Jaw

17. Surgery Deep Bridge Str Jaws

18. Surgery Deep Bridge Delicate

Stille-Giertz Rib Shear

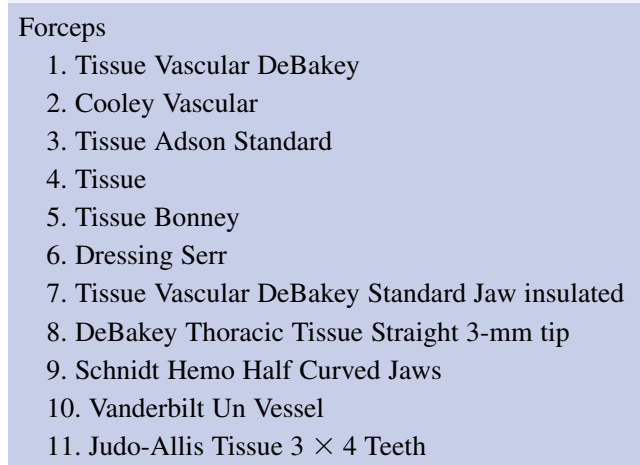

Holders

1. Instrument Single Link

2. Needle Holder

a. Classic Plus Sarot

b. Mayo Hegar Classic Plus

c. Microvasc Classic Plus

d. Classic Plus Crile Wood

e. Mayo Hegar

Scissors

1. Classic Mayo Curved Round Blade

2. Classic Mayo Straight Round Blade 
3. Tonsil Metzenbaum Delicate Curved

4. Classic Plus Metzenbaum Delicate Curved

5. Classic Plus Nelson Delicate Curved

6. Wexler Heavy Thoracic Curved

7. Universal Standard

8. Dissecting $11^{\prime \prime}$ Nelson Vital Curved Regular

Edna Non-Perforating Towel Clip

Knife handle

1. Surgical No. 3 Stainless Steel

2. Surgical No. 3L Stainless Steel

3. Surgical No. 7 Stainless Steel

Harken Rib Spreader-Frame, Arm, Crank, and Blade

Matson Rib Double-Ended Elevator and Stripper Raspatory

Pin Heavy Cutter

Stille-Luer-Type D/A 10MM Curved Jaws

Bailey Rib Contractor

Coastal Alexander Periosteotome- Sharp

Doyen Costal Elevator- Cobb, Right, and Left Blade, Heavy Duty Round Handle

Andrews-Pynchon Suction Tube

Handpiece Interpulse w/Coaxial High Flow

Aquamantys 6.0 Bipolar Sealer

Argon Beam Coagulator

ProTack 5-mm Fixation Device

LIGACLIP Endoscopic Rotating Multiple Clip Applier

Suture Passer 10-12 mm guide

Dual Mesh $20 \mathrm{~cm} \times 30 \mathrm{~cm} \times 1.0 \mathrm{~mm}$

Endo GIA Ultra Universal Stapler

Sutures

1. ETHIBOND 0 CT-1 30

2. Silk $0 \mathrm{MH}$

3. VICRYL 1TP-1

4. VICRYL 0 UR-6

5. VICRYL 2-0 CT-1

6. VICRYL 3-0

7. MONOCRYL 4-0 PS-2 